\title{
On the nature of the sources of the cosmic infrared background.
}

\author{
A. Kashlinsky ${ }^{1,2,4}$, R. G. Arendt ${ }^{1,2}$, J. Mather ${ }^{1,3}$, S. H. Moseley ${ }^{1,3}$
}

\begin{abstract}
We discuss interpretation of the cosmic infrared background (CIB) anisotropies detected by us recently in the Spitzer IRAC based measurements. The fluctuations are approximately isotropic on the sky consistent with their cosmological origin. They remain after removal of fairly faint intervening sources and must arise from a population which has a strong CIB clustering component with only a small shot-noise level. We discuss the constraints the data place on the luminosities, epochs and mass-to-light ratios of the indvidual sources producing them. Assuming the concordance $\Lambda$ CDM cosmology the measurements imply that the luminous sources producing them lie at cosmic times $<1$ Gyr and were individually much brighter per unit mass than the present stellar populations.
\end{abstract}

Subject headings: cosmology: theory - cosmology: observations - diffuse radiation - early Universe

\section{Introduction}

If the early Universe contained significantly more luminous populations than at present, such as is thought to be the case with the very first metal-free stars (see review by Bromm \& Larson 2005), these populations could have produced a significant contribution to the cosmic infrared background (CIB) with potentially measurable structure (Santos et al 2002, Salvaterra \& Ferrara 2003, Cooray et al 2004, Kashlinsky et al 2004; see Kashlinsky (2005) for recent review). In an attempt to uncover the CIB fluctuations from early populations we have analyzed deep images obtained with the Spitzer IRAC instrument (Kashlinsky, Arendt, Mather \& Moseley 2005; hereafter KAMM1), which led to detecting significant CIB fluctuations remaining after subtracting sources to faint flux levels. In a companion paper we

\footnotetext{
${ }^{1}$ Observational Cosmology Laboratory, Code 665, Goddard Space Flight Center, Greenbelt MD 20771

${ }^{2} \mathrm{SSAI}$

${ }^{3}$ NASA

${ }^{4}$ e-mail: kashlinsky@stars.gsfc.nasa.gov
} 
presented analysis from deeper and larger fields using the GOODS Spitzer data (Kashlinsky, Arendt, Mather \& Moseley 2006; hereafter KAMM2), which confirms our earlier findings and extends them to fainter levels of removed galaxy populations and larger angular scales.

In this Letter we discuss the cosmological implications of the recent measurements of the CIB fluctuations from early populations obtained by us (KAMM1,KAMM2). These measurements imply that the signal must come from cosmic sources which have a significant clustering component, but a low shot-noise contribution to the power spectrum. Given the amplitude of the CIB flux expected from these populations in the concordance $\Lambda$ CDM cosmology $\left(\gtrsim 1 \mathrm{nW} / \mathrm{m}^{2} / \mathrm{sr}\right)$, we show that these sources must have very faint individual

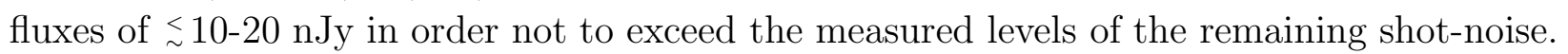
Furthermore, these populations had to have mass-to-light ratio significantly below that of the present day stellar populations in order to produce the required CIB fluxes in the short cosmic time available $(<1 \mathrm{Gyr})$ from the available baryons. Finally, we discuss the prospects for their individual detection with future space missions. We use the AB magnitude system, so flux per frequency $\nu$ of magnitude $m$ is $S_{\nu}(m)=3631 \times 10^{-0.4 m} \mathrm{Jy}$; diffuse flux in $\mathrm{nW} / \mathrm{m}^{2} / \mathrm{sr}$ is defined as $\nu I_{\nu}$, with $I_{\nu}$ being the surface brightness in $\mathrm{MJy} / \mathrm{sr}$.

\section{Magnitudes and epochs of the sources of the CIB fluctuations}

In their analysis KAMM1 and KAMM2 used a total of 5 different fields with deep Spitzer IRAC observations of up to 24 hours per pixel. All the observed fields are located at high Galactic and Ecliptic latitudes and are free of significant zodiacal emissions at all IRAC channels and of cirrus at the IRAC channels 1-3 $(3.6$ to $5.8 \mu \mathrm{m})$. Individual galaxies and other sources were removed until a fixed level of the shot noise from remaining sources was reached. The power spectrum of the remaining diffuse emission showed a residual shot-noise component on small angles and a significant excess due to clustering of faint/distant sources at scales $\gtrsim 0.5^{\prime}$. Within the errors all fields cleaned to the same shot-noise level showed the same excess fluctuations consistent with their cosmological origin (see Fig. 1 of KAMM2). At $8 \mu \mathrm{m}$ there is a significant pollution by the Galactic cirrus and at $5.8 \mu \mathrm{m}$ the larger instrumental noise leads to relatively large errors in the large-scale fluctuations. Here we concentrate on the interpretation of the data at 3.6 and $4.5 \mu \mathrm{m}$ in terms of the luminosities, the epochs and the nature of the cosmological sources contributing to these fluctuations.

KAMM1-2 show that the CIB fluctuations must come from cosmological sources, such as ordinary galaxies and the putative Population III. The former are defined as metal-rich stars with IMFs of a Salpeter-Scalo (Kennicutt 1998) type with masses $\sim 1 M_{\odot}$. Population III is defined (loosely) as luminous sources that existed at, say, $z_{\sim}^{>10}$ which possibly were individu- 
ally very massive and intrinsically very luminous. Data such as discussed here cannot resolve whether the sources contributing to the CIB were metal-rich (Fernandez \& Komatsu 2005) and whether the source of this radiation was stellar nucleosynthesis (Santos et al 2002) or black-hole accretion in the early Universe (Cooray \& Yoshida 2004). Population III epochs, $z>10$, may contain emissions by both stars and quasar-like objects (Kashlinsky \& Rees 1983).

Any model aimed to explain the CIB fluctuations results must reproduce three major aspects: 1) The sources producing the measured CIB fluctuations must be fainter than those removed from the data. 2) They must reproduce the observed excess CIB fluctuations at $\gtrsim 0.5^{\prime}$, where $\delta F \simeq 0.07-0.1 \mathrm{nW} / \mathrm{m}^{2} / \mathrm{sr}$. 3) Lastly, the populations below the above cutoff must account not only for the correlated part of the CIB, but must also reproduce the observed (low) shot-noise component of the signal. These lead to:

1) The the shot-noise component of the power spectrum from source counts $d N / d m$ per magnitude interval $d m$ is $P_{\mathrm{SN}}=\int S^{2}(m) d N(m)$ with $S=\nu S_{\nu}$. To estimate limiting magnitudes implied by the measured shot-noise, we generated source counts for the observed fields with SExtractor (Bertin \& Arnouts 1996). Fig. 1 shows the remaining shot noise levels in KAMM1-2 analysis and the counts data. The intersection of the counts with the lowest shot noise levels shows that the sources are eliminated to $m_{\sim} 25$-26, so the detected CIB fluctuations come from fainter sources. This magnitude limit at $3.6 \mu \mathrm{m}$ corresponds to only $10^{9} h^{-2} 10^{-0.4(m-25.5)} L_{\odot}$ emitted at $6000 \AA$ at $z=5$ where $h$ is the Hubble constant in units of $100 \mathrm{~km} / \mathrm{sec} / \mathrm{Mpc}$. If the counts contain extra populations in addition to those from (Fazio et al 2004), the magnitude limit will be fainter. Thus KAMM1,KAMM2 have removed a significant fraction of galaxies even at $z=5$ and the CIB fluctuations must come from sources at higher $z$.

2) The clustering component of the CIB at $0.5^{\prime} \underset{\sim}{\sim} 2 \pi / q_{\sim}^{<} 5^{\prime}$ requires $F_{\mathrm{CIB}} \sim$ a few $\mathrm{nW} / \mathrm{m}^{2} / \mathrm{sr}$ as noted by us earlier (KAMM1). The rms fluctuation in the CIB flux, $\delta F=\sqrt{q^{2} P_{2}(q) / 2 \pi}$, on angular scale $2 \pi / q$ is related to the underlying 3-dimensional power spectrum of the emitters' clustering, $P_{3}(k)$, the duration over which the flux was produced, $\Delta t$, and the rate of the CIB production rate, $d F / d t$, via the Limber equation (e.g. Kashlinsky 2005a):

$$
\delta F=F_{\mathrm{CIB}} \bar{\Delta}_{F} ; \bar{\Delta}_{F}^{2} \equiv \frac{\Delta t \int_{\Delta t}(d F / d t)^{2} \Delta^{2}\left(q d_{A}^{-1}\right) d t}{\left[\int_{\Delta t}(d F / d t) d t\right]^{2}}
$$

where $\Delta(k)=\left[k^{2} P_{3}(k) / 2 \pi c \Delta t\right]^{1 / 2}$ is the rms fluctuation in source counts over the cylinder of radius $2 \pi / k$ and length $c \Delta t$. In the limit when the CIB release rate is approximately constant, the relative CIB fluctuation, $\bar{\Delta}_{F}$, will be $\sim\left\langle\Delta^{2}\left(q d_{A}^{-1}\right)\right\rangle^{1 / 2}$ with $\langle\ldots\rangle \equiv(\Delta t)^{-1} \int_{\Delta t} \ldots d t$. If $d F / d t$ peaks at some cosmic epoch $z_{p}$, the relative fluctuation will be $\simeq \Delta\left(q d_{A}^{-1}\left(z_{p}\right)\right)$.

To evaluate the range of the expected CIB flux from the sources producing the mea- 
sured fluctuations, we adopt the $\Lambda$ CDM model with $\left(\Omega, \Omega_{\text {baryon }}, \Omega_{\Lambda}, h\right)=(0.3,0.044,0.7,0.71)$ and consider the epochs spanning $5 \leq z \leq 20$. The cosmic time at $z=20$ is $\simeq 0.2$ Gyr and the time between $z=20$ and $z=5$ is $1 \mathrm{Gyr}$. The scale $r_{8}=8 h^{-1} \mathrm{Mpc}$, with today's density contrast $\sigma_{8}$, subtends $\theta_{8} \simeq(3-4)^{\prime}$. The relative fluctuation in the projected 2-dimensional power spectrum, $\Delta$, on that angular scale $\theta_{8}$, produced from sources located at mean value of $\bar{z}$ and spanning the cosmic time $\Delta t$, would be $\Delta\left(\theta_{8}\right) \sim \sigma_{8}(1+\bar{z})^{-1}\left(r_{8} / c \Delta t\right)^{1 / 2} \simeq$ $0.02 \sigma_{8}\left(\frac{\bar{z}}{10}\right)^{-1}\left(\frac{\Delta t}{\text { Gyr }}\right)^{-1 / 2}$ neglecting the amplification due to biasing. Biasing, due to sources forming out of rare peaks of the density field, will increase $\Delta$ (Kaiser 1984) and for reasonable bias factors $\left(\sim 2\right.$ for systems collapsing at $z \sim 5$ to $>3$ at $z_{\sim}^{>10}$ ) one can gain amplification factors, $A$, in $\Delta$ of $\simeq 2$ to $\approx 4-5$ between $z=5$ and 20 (Kashlinsky 1991; Kashlinsky 1998; Cooray et al 2004; Kashlinsky et al 2004). Thus the arcminute scale CIB fluctuations of $\delta F \sim 0.07-0.1 \mathrm{nW} / \mathrm{m}^{2} / \mathrm{sr}$ at $3.6,4.5 \mu \mathrm{m}$ require the mean CIB from these sources to be $F_{\mathrm{CIB}} \sim 4-5\left\langle A[(1+z) / 6]^{-1}\right\rangle^{-1}(\Delta t / 1 \mathrm{Gyr})^{1 / 2} \mathrm{nW} / \mathrm{m}^{2} / \mathrm{sr}$. Assuming that the fluctuations are produced by low surface brightness systems at much lower $z$ does not alter the required high value of their mean $\mathrm{CIB}$ contribution: e.g. taking $\Delta t=5 \mathrm{Gyr}$ corresponding to the cosmic time between $z=1$ and 20 gives $\bar{\Delta} \simeq 0.02$ at $1^{\prime}$ assuming no biasing. (As discussed below such sources would likely produce shot-noise in excess of what we measure.) We can reach similar conclusions with the entire range of scales $\underset{\sim}{>} 0.5^{\prime}$ where we measure the clustering component of the CIB. The left panels of Fig. 1 show the least squares fits for $F_{\mathrm{CIB}}$, assuming the $\Lambda \mathrm{CDM}$ model, from all the fields data at 3.6 and $4.5 \mu \mathrm{m}$. This gives $F_{\mathrm{CIB}}\left\langle A\left(\frac{1+z}{10}\right)\right\rangle_{\sim}(4,2.5)(\Delta t / 1 \mathrm{Gyr})^{-1 / 2} \mathrm{nW} / \mathrm{m}^{2} / \mathrm{sr}$ at $(3.6,4.5) \mu \mathrm{m}$ respectively.

We thus conservatively take the fiducial flux of $F_{\mathrm{CIB}}=1 \mathrm{nW} / \mathrm{m}^{2} / \mathrm{sr}$ as the minimal CIB flux at 3.6 and $4.5 \mu \mathrm{m}$ required by the fluctuations, corresponding to the relative minimal CIB fluctuations of $\sim 7 \%$. The results below can be re-scaled to arbitrary $F_{\mathrm{CIB}}$, but our general conclusions will be valid unless the CIB flux from sources producing the measured fluctuations is significantly below the above number. Although the net CIB fluxes may be, in principle, much higher, this minimal CIB level at $3.6 \mu \mathrm{m}$ is smaller than the claimed CIB excess from DIRBE and IRTS measurements over that from galaxy counts (Dwek \& Arendt 1998; Arendt \& Dwek 2003; Matsumoto et al 2005), and is consistent with the recent measurements of absorption in the spectra of fairly distant $(z=0.13-0.18)$ blazars at TeV energies (Dwek et al 2005; Aharonian et al 2006). Such CIB levels should, however, be measurable from the spectra of gamma-ray bursts at $z_{\sim}^{>1-2}$ detectable with the upcoming NASA's GLAST mission out to 300 Gev (Kashlinsky 2005b). Spitzer counts (Fazio et al 2004) show that the remaining ordinary galaxies can contribute only $\simeq 0.15$ $\mathrm{nW} / \mathrm{m}^{2} / \mathrm{sr}$ at $3.6 \mu \mathrm{m}$ (KAMM1), so to explain the CIB fluctuations with the remaining (extrapolated) Spitzer counts sources requires almost $\sim 100 \%$ fluctuation on arcminute scales.

3) The CIB in the populations producing the measured fluctuations significantly ex- 
ceeds that from extrapolated IRAC counts (Fazio et al 2004), so the excess flux must come from fainter populations with a significant deviation from the extrapolated counts slope (KAMM1). The measured fluctuations indicate a population with a relatively strong clustering component, which at the same time has low shot noise. This means that the sources must be individually faint. The shot-noise from the remaining galaxies dominates the power spectrum of the CIB at $\lesssim 0.5^{\prime}$ and its amplitude sets an upper limit on the shot-noise component of the sources contributing to the arcminute scale CIB fluctuations. The amplitude of the shot-noise component is (Kashlinsky 2005a): $P_{\mathrm{SN}}=\int_{>m} S(m) d F(m) \equiv S(\bar{m}) F_{\text {tot }}(>m)$, where $d F(m)=S(m) d N(m)$ is the CIB from sources at the magnitude interval $d m$ and $F_{\text {tot }}(m)$ is the total flux from the remaining sources of $>m$. The sources contributing to the clustering component of the fluctuations at arcminute scales must not exceed the level of the residual shot noise in the data of $P_{\mathrm{SN}} \simeq(2,1) \times 10^{-11} \mathrm{nW}^{2} / \mathrm{m}^{4} / \mathrm{sr}$ at $(3.6,4.5) \mu \mathrm{m}$. At $4.5 \mu \mathrm{m}$ this shot-noise amplitude of $P_{\mathrm{SN}}=10^{-11} \mathrm{nW}^{2} / \mathrm{m}^{4} / \mathrm{sr}$ or $10(\lambda / 3 \mu \mathrm{m})^{-1} \mathrm{nJy} \cdot \mathrm{nW} / \mathrm{m}^{2} / \mathrm{sr}$, would lead to sources contributing to the signal having mean fluxes less than $12\left(F_{\mathrm{CIB}} / \mathrm{nWm}^{-2} \mathrm{sr}^{-1}\right)^{-1}$ nJy or $\mathrm{AB}$ magnitudes $\bar{m} \geq 29+2.5 \lg \left(\frac{F_{\mathrm{CIB}}}{\mathrm{nWm}^{-2} \mathrm{sr}^{-1}}\right)$. At $3.6 \mu \mathrm{m}$ the shot-noise levels are a factor of $\simeq 2$ larger leading to $\bar{m}$ about one magnitude brighter. An important further information could be obtained in still deeper measurements by setting a lower limit on the shot-noise component of the sources contributing to the CIB fluctuations determined when the clustering component disappears of is substantially reduced.

\section{Discussion}

More information on the nature of populations of these faint sources can be obtained by considering the fraction of baryons that went through stars prior to $z_{\sim}^{>} 5\left(\Delta t_{\sim}^{<}\right.$Gyr) needed to explain the level of the CIB required by our data. The net flux at frequency $\nu$ produced by the population with comoving luminosity density $\mathcal{L}$, is $F_{\mathrm{CIB}}=\frac{c}{4 \pi} \int_{\Delta t} \mathcal{L}_{\nu^{\prime}}(1+z)^{-1} d t$, where $\nu^{\prime}=\nu(1+z)$. This requires the average comoving luminosity density at $(0.36-0.45) \mu \mathrm{m} \frac{10}{1+z}$ of:

$$
\overline{\mathcal{L}} \simeq \frac{4 \pi}{c} F_{\mathrm{CIB}}(\Delta t)^{-1}(1+\bar{z}) \simeq 1.2 \times 10^{9} L_{\odot} \mathrm{Mpc}^{-3} \frac{1 \mathrm{Gyr}}{\Delta t} \frac{1+\bar{z}}{10} \frac{F_{\mathrm{CIB}}}{\mathrm{nWm}^{-2} \mathrm{sr}^{-1}}
$$

For comparison the present-day luminosity density measured by the Sloan Digital Sky Survey at $0.32 \mu \mathrm{m}$ to $0.68 \mu \mathrm{m}$ is about an order of magnitude lower (Blanton et al 2003). This indicates significantly more luminous populations contributing to the CIB fluctuations than at present. The contribution to the density parameter by these sources is thus given by:

$$
\Omega_{*}=\frac{\left.(\Gamma \overline{\mathcal{L}})\right|_{(0.36-0.45) \mu \mathrm{m} \frac{10}{1+z}}}{3 H_{0}^{2} / 8 \pi G} \simeq 8.3 \times 10^{-3} \frac{F_{\mathrm{CIB}}}{\mathrm{nWm}^{-2} \mathrm{sr}^{-1}} \frac{\Gamma}{\Gamma_{\odot}}\left(\frac{\Delta t}{1 \mathrm{Gyr}}\right)^{-1} \frac{1+\bar{z}}{10}
$$


where $\Gamma$ is the mass-to-light ratio. For comparison the mean density in present day stars is significantly lower at $\Omega_{*, z=0} \simeq 2 \times 10^{-3}$ (Fukugita et al 1998; Cole et al 2001) and much of the contribution to $\Omega_{*, z=0}$ comes from the late stellar Population I stars with solar metallicities. Strictly speaking eq. 3 assumes no re-processing of baryons and may thus overestimate the required amount of luminous baryons in the case of short lived massive stars, such as Population III, but it shows that it is energetically easier to produce the significant CIB levels implied by the Spitzer data in the cosmic time available with stars whose mass-function is skewed toward $\Gamma \ll \Gamma_{\odot}$. (For populations made up of massive stars it can be replaced with eq. 3 of Kashlinsky, 2005b). If the CIB fluctuations are produced by populations containing a significant fraction of low-mass stars, which should still be burning today, they would require a large fraction of the present-day stars to have been produced at $z \geq 6-10$.

To model the ordinary stellar populations, we have run stellar evolution models using the PEGASE code (Fioc \& Rocca-Volmerange 1998), assuming normal IMF with various metallicities and the ongoing star formation (i.e. star formation rate $\propto \exp \left(-t / t_{\text {burst }}\right)$ with $t_{\text {burst }}=20 \mathrm{Gyr}$ ). For Population III we adopted the spectral energy distribution from (Santos et al 2002). Fig. 2 shows the luminosity per unit mass in stars $\left(\Gamma^{-1}\right)$ assuming the ordinary population to be less than 1 Gyr old $\left(\Gamma \sim 0.2-0.5 \Gamma_{\odot}\right)$ and contrasts them with the expectations for massive Pop III systems $\left(10^{-2}-10^{-3} \Gamma_{\odot}\right)$. If the CIB fluctuation signal comes entirely from the Population III systems, eq. 3 would give the minimal fraction of baryons locked in them $\sim 0.15 \%\left(F_{\mathrm{CIB}} / \mathrm{nW} \mathrm{m}^{-2} \mathrm{sr}^{-1}\right)$. If the baryons are re-used in stars this fraction would be decreased. This number is in agreement with that of (Kashlinsky 2005b) after scaling to the appropriate CIB levels: $0.14 \%\left(F_{\mathrm{CIB}, \text { bolometric }} / \mathrm{nW} \mathrm{m}^{-2} \mathrm{sr}^{-1}\right)(z / 10)(\epsilon / 0.007)^{-1}$ assuming the hydrogen burning efficiency $\epsilon$. (Such massive stars would be fully convective with the overall efficiency reaching $\epsilon_{\sim}^{>3} \times 10^{-3}$, Schaerer 2002).

The sources satisfying the above constraints had masses in luminous matter of:

$$
M_{*} \sim 4 \pi d_{L}^{2}(1+z)^{-1} \Gamma S(\bar{m})_{\sim}^{<} 7 \times 10^{5} h^{-2} M_{\odot} \frac{\Gamma_{(3.6-4.5) \mu \mathrm{m}}}{5 \times 10^{-3} \Gamma_{\odot}} \frac{[S(\bar{m}) / \nu]}{20 \mathrm{nJy}}\left(\frac{1+z}{10}\right)^{1.6}
$$

where the luminosity distance was approximated $d_{L} \simeq 3.2(1+z)^{1.3} h^{-1} \mathrm{Gpc}$. Such Population III sources, with only $\lesssim$ a few times $10^{5} M_{\odot}$ in stellar material, would be below the detection threshold in the high- $z$ Lyman dropout searches of (Bouwens et al 2005; Willis \& Courbin 2005) considered by (Salvaterra \& Ferrara 2006). In any case theoretical predictions of the luminosity function of Population III sources are necessarily modeldependent as they depend on the assumptions of the small-scale power and its evolution as well as the microphysics governing the various feedback effects during the collapse of the first haloes. The Press-Schechter type prescriptions may break down for the slope and regime of power spectra on the relevant scale (Springel et al 2005) and the feedback mechanisms 
due to the $H_{2}$ destruction by the Lyman-Werner bands radiation (Haiman et al 1997) likely suppress star formation in a complicated halo-mass dependent way.

To resolve the faint sources responsible for the CIB fluctuations, their individual flux must exceed the confusion limit usually taken to be $\alpha \geq 5$ times the flux dispersion produced by these emissions (Condon 1974). Lower flux sources will be drowned in the confusion noise; of course, this is precisely where CIB studies would take off. If such sources were to contribute the CIB required by our data, at $3.6 \mu \mathrm{m}$ they had to have the average surface density of $\bar{n} \sim F_{\mathrm{CIB}}^{2} / P_{\mathrm{SN}} \sim 2 \operatorname{arcsec}^{-2}\left(\frac{F_{\mathrm{CIB}}}{\mathrm{nWm}^{-2} \mathrm{sr}^{-1}}\right)^{2}\left(\frac{P_{\mathrm{SN}}}{10^{-11} \mathrm{nW}^{2} \mathrm{~m}^{-4} \mathrm{sr}^{-1}}\right)^{-1}$. To avoid the confusion limit and resolve these sources individually at, say, 5-sigma level $(\alpha=5)$ one would

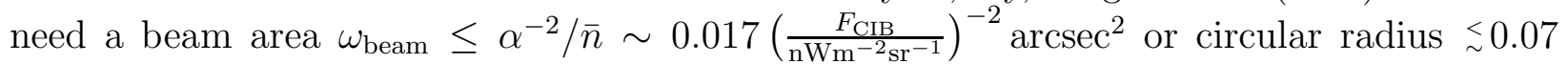
$\left(F_{\mathrm{CIB}} / \mathrm{nWm}^{-2} \mathrm{sr}^{-1}\right)^{-1}$ arcsec. This is not in the realm of the current instruments, but the JWST could be able to resolve these objects (Gardner et al 2006). Extrapolation of this argument to shorter $\lambda$ is model-dependent as it would assume both the SED of these sources (to predict their magnitudes at $\lambda<3 \mu \mathrm{m}$ ) and their $z$ (to predict the location of their Lyman break and whether or not they are observable at $\lambda<3 \mu \mathrm{m}$ ). In any case, at 1.1 and $1.6 \mu \mathrm{m}$ confusion is not reached until $m_{\mathrm{AB}}>28$ (Thompson et al 2005). If the first stars produced dusty environments their far-IR luminosities will be substantial and these sources should be visible at wavelengths redshifted today to $\mathrm{mm}$ and sub-mm bands. In that case, they may be resolvable with the ALMA 1 large array, whose sub-mm resolution is better than $0.02^{\prime \prime}$.

Finally, the fluctuations are unlikely to come from low-luminosity low- $z$ normal galaxies. Such galaxies must have the surface density $\bar{n}_{\sim}^{>} 3 \times 10^{7} \mathrm{deg}^{-2}$ with the $3.6,4.5 \mu \mathrm{m}$ fluxes $\underset{\sim}{<10-20}$ nJy. Unless they are significantly fainter than this limit, emissions from star-forming systems should have comparable flux at shorter $\lambda$ out to the $4000 \AA$ break for passively evolving populations or to the Lyman cutoff at $\simeq 0.1 \mu \mathrm{m}$ for star-forming galaxies. Galaxy counts now extend to $m \simeq 30.5$ (2 nJy) at $0.67 \mu \mathrm{m}$ and to 29 (10 nJy) at $1.6 \mu \mathrm{m}$ (Madau \& Pozzetti 2000) and are over an order of magnitude below the required value of $\bar{n}$ at the faintest magnitudes. This would exclude star forming galaxies as faint as 2 nJy at $0.67 /(1+z) \mu \mathrm{m}$ at $z \approx 5.7$ and passively evolving populations out to $10 \mathrm{nJy}$ at $1.6 /(1+z) \mu \mathrm{m}$ at $z_{\sim}^{<3}$. We note, however, that this analysis cannot exclude "abnormal" populations at low $z$.

This work is supported by NSF AST-0406587 and NASA Spitzer NM0710076 grants.

\footnotetext{
${ }^{1}$ http://www.alma.nrao.edu/
} 


\section{REFERENCES}

Aharonian, F. et al 2006, Nature, 440,1018

Arendt, R. \& Dwek, E. 2003, Ap.J., 585, 305

Bertin, E. \& Arnouts, S. 1996, Astron.Astrophys. Suppl., 117, 393-404

Blanton, M.R. et al 2003, Ap.J., 592, 819-838

Bouwens, R.J., Illingworth, G., Thompson, R., \& Franx, M. 2005, Ap.J., 624, L5-L8

Bromm, V. \& Larson, R. 2004, Ann. Rev. A. A., 42, 79

Bruzual, A. \& Charlot, S. 2003, MNRAS, 344, 1000-1028

Cooray, A. et al 2004, Ap.J., 606, 611

Cooray, A. \& Yoshida, N. 2004, MNRAS, 351, L71

Cole, S. et al 2001, MNRAS, 326, 255-273

Condon, J. 1974, Ap.J., 188, 279-286

Dickinson, M. et al 2003, "The great observatories origins deeps survey", in "The mass of galaxies at low and high redshift", ed. R. Bender \& A. Renzini, astro-ph/0204213

Dwek, E. \& Arendt, R. 1998, Ap.J.,508,L9

Dwek, E., Krennrich, F., \& Arendt, R. 2005, Ap.J., 634,155

Fazio, G. et al 2004, Ap.J.Suppl., 154, 39

Fernandez, E. \& Komatsu, E. 2005, Ap.J., astro-ph/0508174

Fioc, M. \& Rocca-Volmerange, B. 1998, Astron. Astrophys., 326, 950-962

Fukugita, M., Hogan, C.J. \& Peebles, P.J.E. 1998, 503, 518-530

Gardner, J.P. et al 2006, Space Sci Reviews, in press. astro-ph/0606175

Haiman, Z., Rees, M.J., \& Loeb, A. 1997, Ap.J., 476, 458

Kaiser, N. 1984, Ap.J., 284, L9

Kashlinsky 1991, Ap.J., 376, L5 
Kashlinsky 1998, Ap.J., 492, 1

Kashlinsky, A. 2005, Phys. Rep., 409, 361-438

Kashlinsky, A. 2005, Ap.J., 633, L5

Kashlinsky, A. \& Rees, M.J. 1983, MNRAS, 205, 955

Kashlinsky, A., Arendt, R., Gardner, J.P., Mather, J.C., \& Moseley, S.H. 2004, Ap.J., 608, 1

Kashlinsky, A., Arendt, R., Mather, J.C. \& Moseley, S.H. 2005, Nature, 438, 45. (KAMM1)

Kashlinsky, A., Arendt, R., Mather, J.C. \& Moseley, S.H. 2006, Ap.J., submitted. (KAMM2)

Kennicutt, R. 1998, ARAA, 36,189

Madau, P. \& Pozzetti, L. 2000, MNRAS, 312, L9

Madau, P. \& Silk, J. 2005,MNRAS, 359, L37

Matsumoto, M. et al 2005, Ap.J., 626, 31

Press, W. \& Schechter, P. 1974, Ap.J., 184, 425

Santos, M.R., Bromm, V., Kamionkowski, M. 2002,MNRAS,336,1082

Salvaterra, R. \& Ferrara, A. 2003, MNRAS, 339, 973

Salvaterra,R. \& Ferrara, A. 2006, MNRAS, 367, L11-L15

Savage, R.S. \& Oliver, S. 2005, astro-ph/0511359

Scalo, J.M. 1986, Fundamentals of Cosmic Physics, 11, 1-278

Schaerer, D. 2002, Aston. Astrophys., 382,28

Springel, V. et al 2005, Nature, 435, 629

Thompson, R. I. et al 2005, Astron. J., 130, 1-12

Willis, J.P. \& Courbin, F. 2005, MNRAS, 357, 1348-1356

This preprint was prepared with the AAS IATEX macros v5.2. 

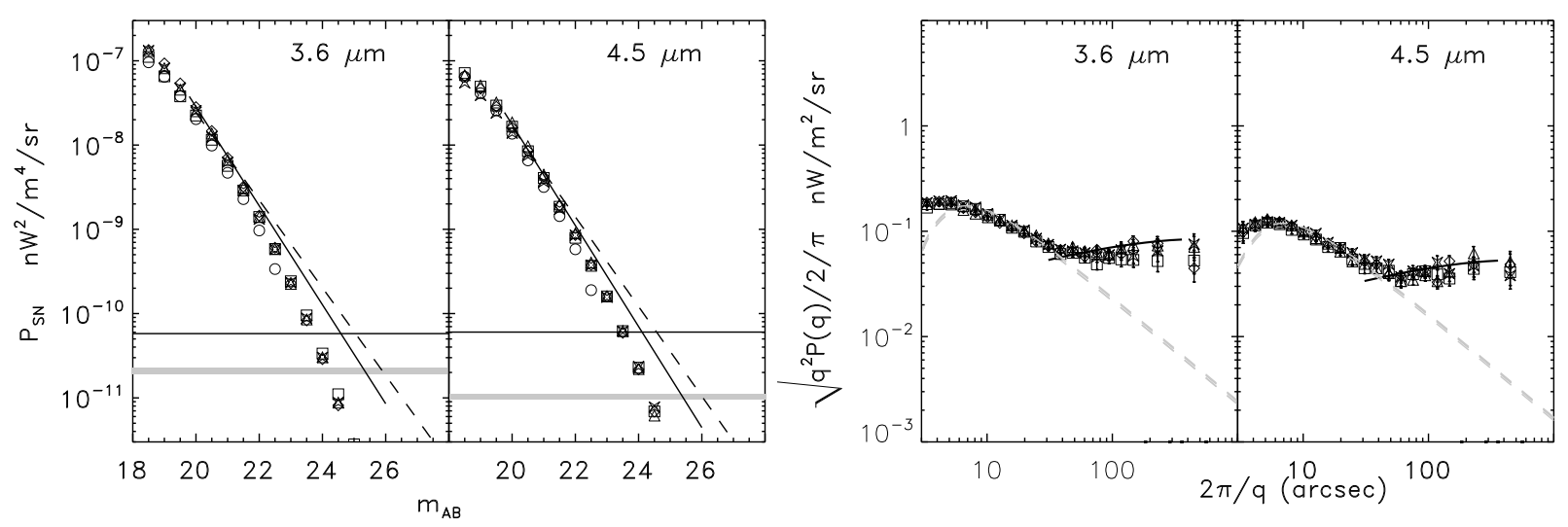

Fig. 1. - Left: Shot noise power amplitude from the data is compared to the values of $P_{\mathrm{SN}}$ estimated by integrating the counts. Solid lines show the levels of $P_{\mathrm{SN}}$ reached in the QSO1700 analysis (KAMM1). Light shaded areas show the levels of $P_{\mathrm{SN}}$ reached in KAMM2. Symbols plot $P_{\mathrm{SN}}$ by integrating the counts evaluated for all five fields in Table 1 of KAMM2. Diamonds correspond to HDFN-E1 region, triangles to HDFN-E2, squares to CDFS-E1 and asterisks to CDFS-E2; open circles correspond to counts for the QS1700 field. Solid line shows $P_{\mathrm{SN}}$ according to the fit to IRAC counts of (Fazio et al 2004) used in (KAMM1); dashed lines correspond to the IRAC counts analysis from (Savage \& Oliver 2005). The counts are significantly incomplete due to confusion at the levels of $P_{\mathrm{SN}}$ reached with our analysis and give a lower limit on the limiting magnitude. Right: CIB fluctuations from KAMM2 at the shot-noise levels shown with shaded regions in the left panel. The notations for the counts from the GOODS data is the same as in the left panels. Light-shaded dashes show the shot noise fluctuations. Solid lines show the least squares fits to the CIB fluctuations from sources at $z_{\sim} 6$ assuming $\Lambda \mathrm{CDM}$ model as described in the text. 


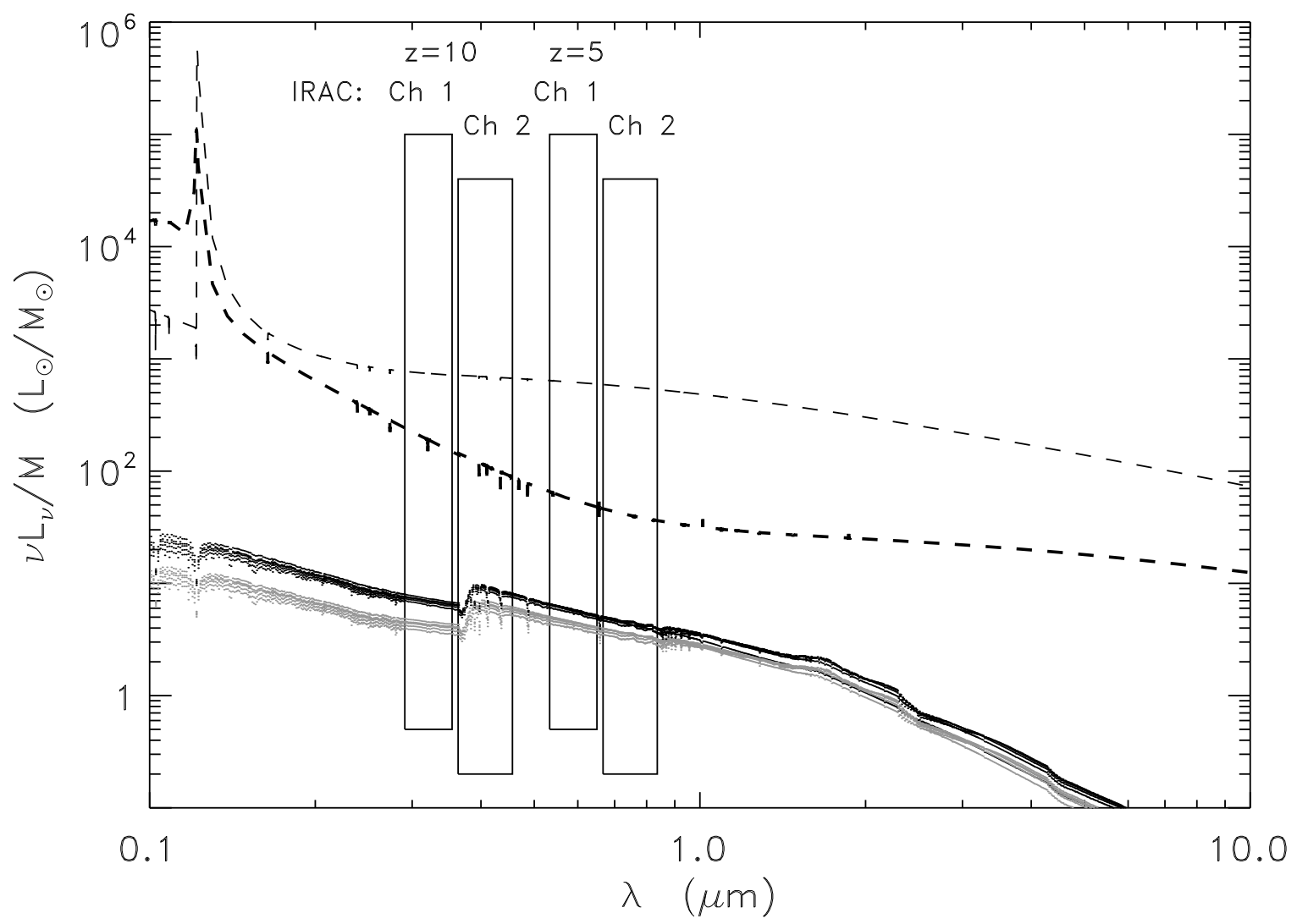

Fig. 2.- Rest-frame luminosity per unit mass plotted vs wavelength for Population III spectra (from Santos et al - dashed lines) and "ordinary" stellar populations at 0.5 and $1 \mathrm{Gyr}$ with Salpeter-Scalo IMF (computed from PEGASE for $Z=0,10^{-3}, 2 \times 10^{-3}, 5 \times 10^{-3}, 10^{-1}$ assuming constant burst of star formation, i.e. $S F R \propto \exp \left(-t / t_{\text {burst }}\right)$ with $t_{\text {burst }}=20$ Gyr.) $L_{\odot}=3.8 \times 10^{33} \mathrm{erg} / \mathrm{sec}$ is the solar bolometric luminosity. The part of emissions probed by the IRAC Channels $1(3.6 \mu \mathrm{m})$ and $2(4.5 \mu \mathrm{m})$ at $z=5,10$ is shown with the marked regions. 